\title{
Odorous and pungent attributes of mixed and unmixed odorants
}

\author{
J. ENRIQUE COMETTO-MUÑIZ \\ Laboratorio de Investigaciones Sensoriales (CONICET-UBA), Buenos Aires, Argentina \\ and John B. Pierce Foundation Laboratory and Yale University, New Haven, Connecticut \\ and \\ SUSANA M. HERNÁNDEZ \\ Laboratorio de Investigaciones Sensoriales (CONICET-UBA), Buenos Aires, Argentina
}

\begin{abstract}
In order to explore functional properties of the olfactory and common chemical senses as well as their relation to the total nasal sensation experienced, various concentrations of two pungent odorants were presented alone and in the presence of different backgrounds of the other irritant. Stimuli comprised formaldehyde (at 1.0, 3.5, 6.9, and $16.7 \mathrm{ppm}$ ), ammonia (at 210, 776, 1,172, and $1,716 \mathrm{ppm}$ ), and their 16 possible binary mixtures. Subjects were asked to estimate the total nasal perceived intensity, and then to assess the olfactory (odor) and common chemical (pungency) attributes of the evoked sensations. The results showed that stimulus-response functions for pungency are steeper than those for odor. Furthermore, odor was always hypoadditive in mixtures (i.e., mixtures were perceived as less intense than the sum of their components), whereas pungency was, mainly, additive, and even suggested hyperadditivity. Total perceived intensity of the stimuli, alone and in mixtures, followed the stimulus-response patterns for pungency, which, therefore, emerged as the dominating attribute used by subjects in scaling the explored range of concentrations. The relationship between total nasal perceived intensity of the mixtures and that of their components reflected hypoaddition, resembling the outcome for the odor attribute.
\end{abstract}

Humans are able to detect airborne chemicals in the environment by means of two sensory systems present in the nose: the olfactory sense and the common chemical sense (CCS). Common chemical sensitivity is not restricted to the nasal mucosa, but extends to the oral mucosa and the conjunctiva. So far as is known, the CCS lacks specialized receptors, which the other chemical sensestaste and smell-have, so the sensations aroused by CCS stimulation are mediated principally by free nerve endings of the trigeminal nerve (Beidler, 1965; Cauna, Hinderer, \& Wentges, 1969). Such sensations comprise irritation, cooling, tingling, prickliness, burning, piquancy, and stinging, among others, all of which can be generically termed pungent sensations. These sensations can arise in the mouth (Cowart, 1987; Dunér-Engström, Fredholm, Larsson, Lundberg, \& Saria, 1986; Green, 1986; Lawless, 1984; Lawless, Rozin, \& Shenker, 1985; Lawless \& D. A. Stevens, 1984, 1988; Rozin, Ebert, \& Schull, 1982; Rozin, Mark, \& Schiller, 1981; Rozin \& Schiller, 1980; Sizer \& Harris, 1985; D. A. Stevens \& Lawless, 1986) as well as in the nose (Cain, 1974, 1976;

This work was supported by a grant to Project 9082-03 from the Consejo Nacional de Investigaciones Científicas y Técnicas (CONICET), República Argentina. Correspondence may be addressed to J. Enrique Cometto-Muñiz, John B. Pierce Foundation Laboratory and Yale University, 290 Congress Avenue, New Haven, CT 06519. J.E.C.-M. is a member of the Carrera del Investigador Científico, CONICET, República Argentina.
Cain \& Murphy, 1980; Cometto-Muñiz \& Cain, 1984; Doty, 1975; Doty et al., 1978; Murphy, 1983). Interestingly, the particular choice of the almost odorless (Cain \& Murphy, 1980) and tasteless carbon dioxide as the CCS stimulant allows the study of both nasal and oral pungency with the same, relatively harmless, substance (ComettoMuñiz \& Cain, 1982; Cometto-Muñiz, García-Medina, Calviño, \& Noriega, 1987; Cometto-Muñiz \& Noriega, 1985; Dunn, Cometto-Muñiz, \& Cain, 1982; GarcíaMedina \& Cain, 1982; J. C. Stevens \& Cain, 1986).

Given that in the real world people face exposure to mixtures of airborne chemicals that are, in most cases, not only odorous but also pungent, the issue of how the olfactory and common chemical senses interact and process these stimuli is of theoretical and practical importance.

A previous study demonstrated that the total nasal perceived intensity of binary mixtures of the pungent odorants formaldehyde $\left(\mathrm{H}_{2} \mathrm{CO}\right)$ and ammonia $\left(\mathrm{NH}_{3}\right)$ showed different degrees of additivity relative to the perceived intensities of the same stimuli when presented alone at the same concentration (Cometto-Muñiz, García-Medina, \& Calviño, 1989). At low concentrations of the stimuli (total range employed: 1.0 to $16.7 \mathrm{ppm}$ for formaldehyde and 210 to $1,716 \mathrm{ppm}$ for ammonia), the total perceived intensity of the mixtures was less than the sum of the perceived intensities of their components (hypoaddition); at intermediate and high concentrations, the total perceived intensity of the mixtures was equal to the sum (simple ad- 
dition) and higher than the sum (hyperaddition), respectively.

These results suggest that the increasing degree of additivity observed as the concentration of the mixed stimuli increases may reflect a progressively greater involvement of the CCS in the total nasal sensation. In other words, at low concentrations, odor may be the most salient feature of the mixtures, whereas at intermediate and high concentrations, pungency may become the salient feature.

This suggestion implies that, if the total nasal perceived intensity of the mixtures is broken down by the subjects into perceived odor and perceived pungency (see Cain, 1976), odor should show hypoaddition, as has been the case in olfactory studies (Berglund, 1974; Berglund, Berglund, \& Lindvall, 1971, 1973; Cain, 1975; Cain \& Drexler, 1974; Foster, 1963; Jones \& Woskow, 1964; Laffort \& Dravnieks, 1982; Laing, Panhuber, Willcox, \& Pittman, 1984; Laing \& Willcox, 1983; Lawless, 1987; Moskowitz \& Barbe, 1977; Patte \& Laffort, 1979; Zwaardemaker, 1907), with very few exceptions (Baker, 1964; Koster, 1969; Rosen, Peter, \& Middleton, 1962), whereas pungency should show simple addition and even hyperaddition.

One of the aims of the present investigation was to test this hypothesis. Another aim was to explore the interaction between odor and pungency, which was previously described to be of mutual inhibition when employing different stimuli for eliciting odor and irritation (pungency) (Cain \& Murphy, 1980).

\section{MATERIALS AND METHOD}

\section{Stimuli}

A two-channel air-dilution olfactometer delivered the various concentrations of each of the two pungent odorants, as well as their mixtures, to the participant's nostril at a flow rate of 4 liters per minute.

Formaldehyde (analytical-grade purity) concentrations were 1.0, $3.5,6.9$, and $16.7 \mathrm{ppm}$, as determined by the chromotropic acid method (NIOSH, 1973).

Ammonia (analytical-grade purity) concentrations were 210, 776, 1,172 , and $1,716 \mathrm{ppm}$, measured spectrophotometrically according to a standard technique (NIOSH, 1974).

There were, then, a total of 24 different stimuli: 4 formaldehyde and 4 ammonia concentrations, plus their 16 binary mixtures.

\section{Subjects}

Twenty-eight subjects (10 males and 18 females) participated. Their average age $( \pm S D)$ was $24.1 \pm 5.2$ years. Males had an average age of $26.1 \pm 6.8$ years, and females $22.9 \pm 3.9$ years.

All participants were nonsmokers, since previous studies showed an impairment in common chemical sensitivity in smokers (ComettoMuñiz \& Cain, 1982; Dunn et al., 1982). The subjects were, mainly, university students or young graduates; at the time of participating in the test, they were in good general health, lacking allergies, colds, or any respiratory-tract disease.

\section{Procedure}

The participants were instructed to use the magnitude estimation method (S. S. Stevens, 1957, 1975), without a prescribed modulus, to judge numerically the total nasal perceived intensity of each stimulus and, then, to split that number for total intensity into two numbers, one reflecting perceived odor intensity and the other reflecting perceived pungency intensity.
Each subject was informed about the meaning of the term pungency; that is, all nasal sensations that are not properly odors: irritation- " "irritación" in Castilian (e.g., the sensation from smelling bleach); burn - "ardor" and "quemazón" in Castilian (e.g., from smelling ammonia); tingle and prickle - "cosquilleo" and "picazón" in Castilian (e.g., from the gas $\mathrm{CO}_{2}$ in soda drinks); sting - "punzadura" in Castilian (e.g., from strong vinegar); and freshness - "frescor" in Castilian (e.g., from menthol).

The subjects assigned any number deemed appropriate to stand for the total nasal perceived intensity of the first stimulus (e.g., $60)$ and then split that number into its component odor intensity (e.g., 40) and its component pungency intensity (e.g., 20). From then on, the participants assigned numbers reflecting perceived intensity (total, odor, and pungency) to each stimulus, using the first stimulus as the standard for comparison (S. S. Stevens, 1957, 1975).

The subjects were told to judge the different sensory qualities on a common scale of perceived magnitude (J. C. Stevens \& Marks, 1980 ). That is, if a perceived odor intensity seemed two times stronger than a perceived pungency, it should be assigned a number twice the one assigned to that pungency.

Stimuli were presented twice each in the course of a test session and in irregular order-that is, in neither monotonic increasing nor decreasing fashion-but not completely at random, since we avoided presenting a very weak stimulus immediately after a very strong one, or vice versa. The second presentation of any stimulus did not occur until all the other stimuli had been presented at least once.

At the beginning of the session, the subjects chose one nostril (the more sensitive or, if both were equally sensitive, the more comfortable to work with) and used that nostril throughout the session.

In each trial, the participants were instructed to inhale for $2.5 \mathrm{sec}$ (paced by a metronome), maintaining the inhalation (or sniffing) effort as constant as possible through the different trials.

\section{Data Analysis}

Because each subject was free to choose his or her own modulus (i.e., to assign any number deemed appropriate to stand for the intensity of the first stimulus), the variability around the mean value for each stimulus was artificially high. To eliminate the scatter due to differences in modulus, the data were normalized to make all subjects' overall geometric means the same (Cain \& Moskowitz, 1974; Lane, Catania, \& S. S. Stevens, 1961).

Data were summarized in terms of the geometric mean of each subject's average (arithmetic mean) response for each stimulus.

\section{RESULTS}

Figure 1 shows the stimulus-response (psychophysical) functions for the total nasal perceived intensity of formaldehyde in the presence of five ammonia backgrounds, including zero (left panel) and of ammonia in the presence of five formaldehyde backgrounds, including zero (right panel).

The results of a two-way ANOVA with interaction performed on the total nasal perceived intensity functions, taking formaldehyde-concentration steps as one factor and ammonia backgrounds as the other factor (Figure 1, left panel) revealed highly significant effects for formaldehyde $[F(3,81)=18.67, p<.01]$ and for ammonia $[F(4,108)$ $=40.33, p<.01]$, as well as a significant interaction $[F(12,324)=2.20, p=.01]$. An analogous ANOVA taking ammonia-concentration steps as one factor and formaldehyde backgrounds as the other (Figure 1, right panel) showed highly significant effects for ammonia $[F(3,81)=40.00, p<.01]$ and formaldehyde $[F(4,108)$ 


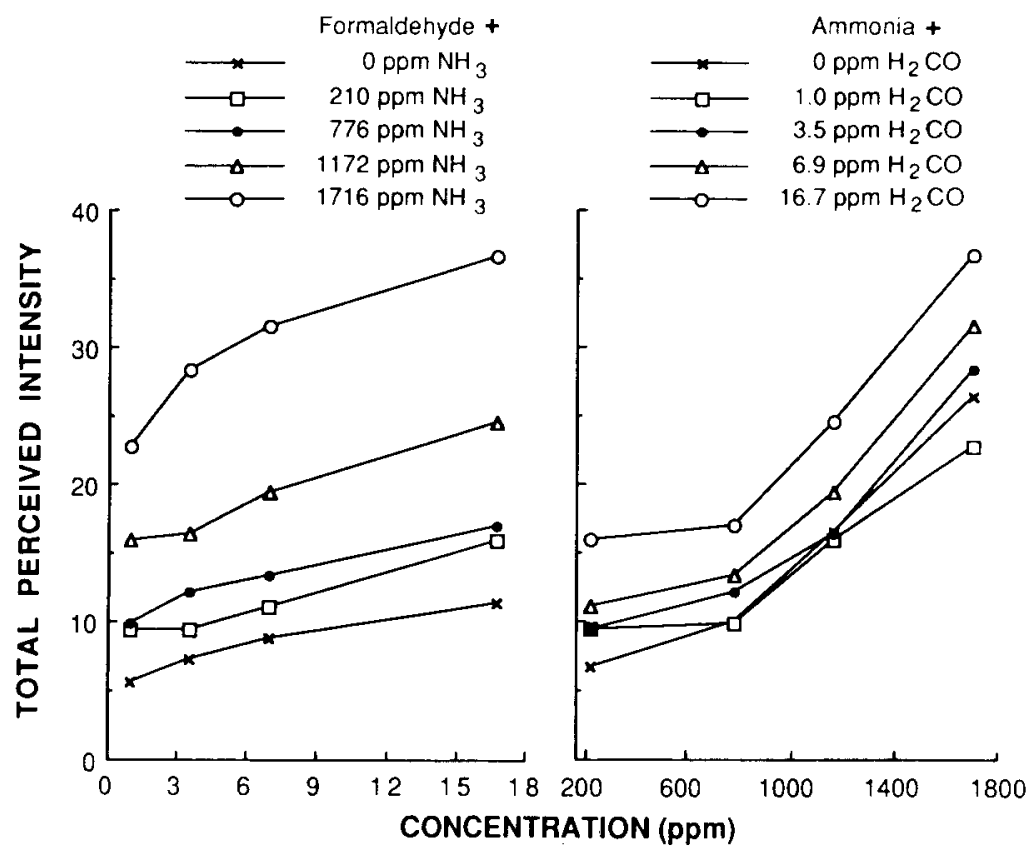

Figure 1. Total nasal perceived intensity as a function of concentration of formaldehyde with ammonia concentration as the parameter (left panel), and as a function of concentration of ammonia with formaldehyde as the parameter (right panel). Each point represents the geometric mean of the average of two replicates made by 28 subjects.

$=16.83, p<.01]$, as well as a significant interaction $[F(12,324)=1.78, p=.05]$. The significance of the interaction term for both groups of functions indicates that functions within each group are not parallel; the figure suggests that they tend to diverge as concentration increases.

Figure 2 depicts the stimulus-response functions for perceived odor intensity of the same stimuli as in Figure 1. A two-way ANOVA with interaction was performed on the odor functions for formaldehyde (left panel) and for ammonia (right panel). Formaldehyde odor functions revealed significant effects only for the ammonia backgrounds $[F(4,108)=3.94, p<.01]$. Ammonia odor functions showed significant effects only for the ammoniaconcentration steps $F(3,81)=4.35, p<.01$ ]

Figure 3 presents the stimulus-response functions for perceived pungency of the same stimuli as in Figure 1. A two-way ANOVA with interaction was performed on the pungency functions for formaldehyde (left panel) and for ammonia (right panel). The results for the formalde-

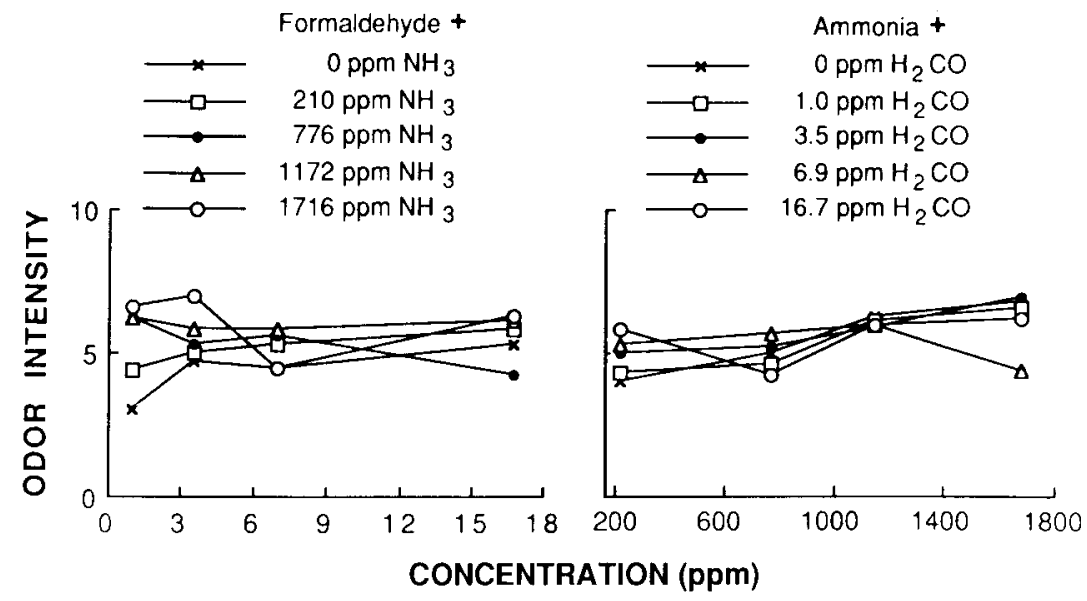

Figure 2. Perceived odor intensity as a function of concentration of formaldehyde with ammonia concentration as the parameter (left panel), and as a function of concentration of ammonia with formaldehyde as the parameter (right panel). Each point represents the geometric mean of the average of two replicates made by 28 subjects. 


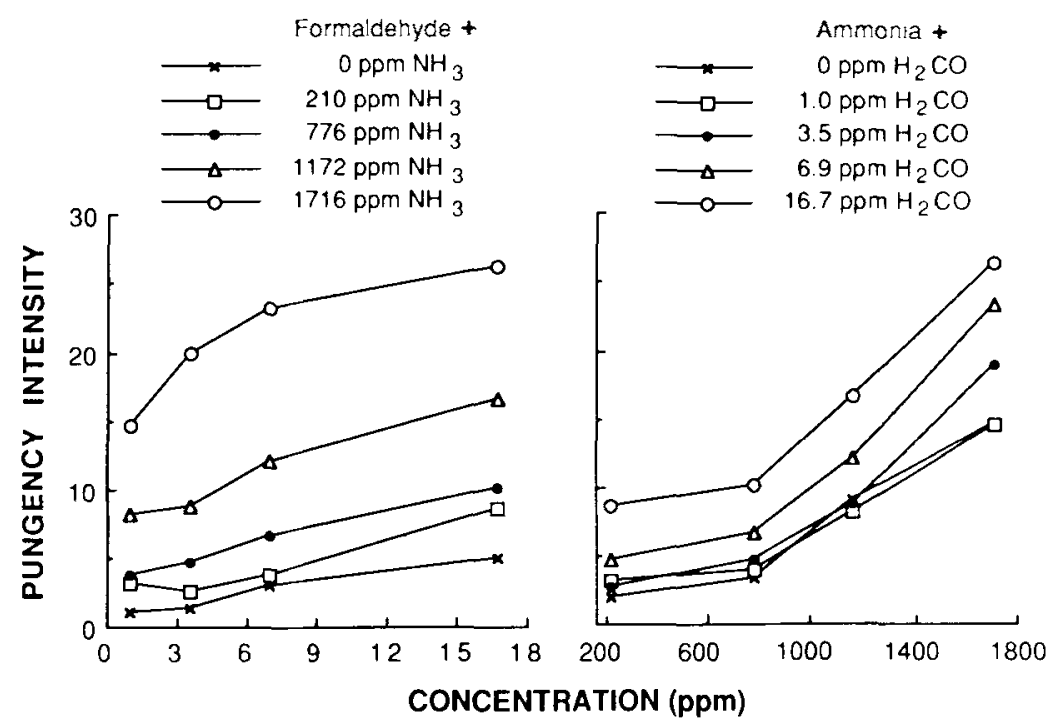

Figure 3. Perceived pungency as a function of concentration of formaldehyde with ammonia concentration as the parameter (left panel), and as a function of concentration of ammonia with formaldehyde as the parameter (right panel). Each point represents the geometric mean of the average of two replicates made by 28 subjects.

hyde functions revealed highly significant effects for the formaldehyde-concentration steps $[F(3,81)=24.67$, $p \ll .01]$ and for the ammonia backgrounds $[F(4,108)=$ $43.63, p<.01]$, and a significant interaction $[F(12,324)$ $=2.83, p<.01]$. Similarly, the outcome for the ammonia functions showed highly significant effects for the ammonia-concentration steps $[F(3,81)=48.17, p<.01]$ and for the formaldehyde backgrounds $[F(4,108)=$ $21.12, p<.01]$, and a significant interaction $[F(12,324)$ $=2.71, p<.01]$. The significant interaction indicates that pungency functions are not parallel; Figure 3 suggests that they tend to diverge with increasing concentration.

Figures 1, 2, and 3 reveal that, for both formaldehyde and ammonia, separately and combined, pungency is the attribute that governs the shape of the psychophysical functions when scaling total nasal perceived intensity. By contrast, odor intensity functions are almost completely flat. Given that total nasal intensity significantly increased with concentration, the flatness of the odor functions indicates that odor makes a much more important relative contribution to total intensity at low than at intermediate or high concentrations. The fact that only the ammonia factor is significant when analyzing the odor functions (Figure 2) reveals that, over the concentration range explored, ammonia evoked a slightly increasing odor while formaldehyde did not.

Figure 4A depicts the relationship between the total nasal perceived intensity of each mixture ( $\Psi$ mixture) and the sum of the perceived intensities of its components

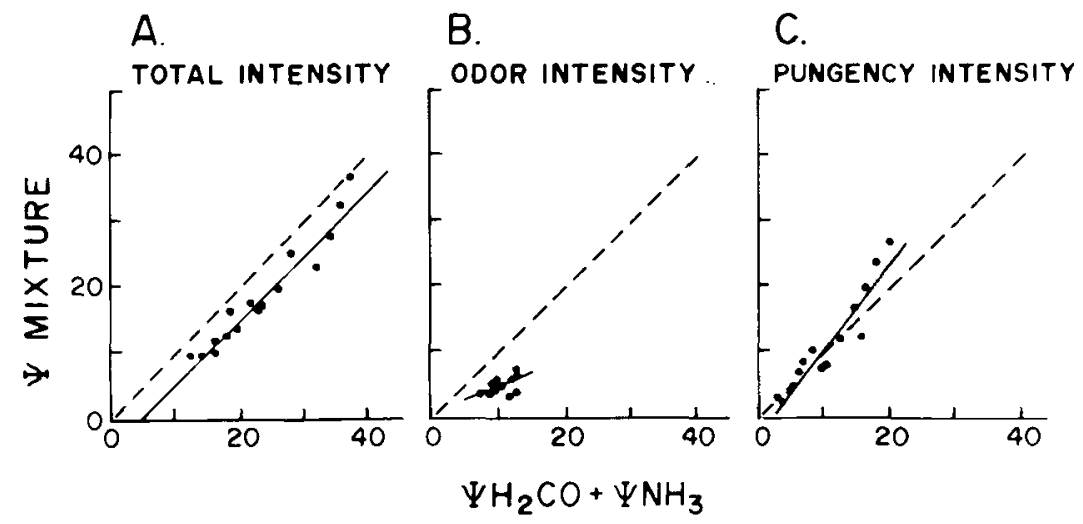

Figure 4. Relationship between the perceived intensity of each of the 16 binary mixtures for (A) total nasal intensity, (B) odor, and (C) pungency; and the sum of the perceived intensities of the components of each mixture for that same attribute. Best-fitted straightline equations and correlation coefficients are: (A) $y=1.00 x-4.78, r=0.97$; (B) $y=$ $0.29 x+2.69, r=0.50$; and (C) $y=1.30 x-2.07, r=0.97$. 
$\left(\Psi \mathrm{H}_{2} \mathrm{CO}+\Psi \mathrm{NH}_{3}\right)$ evaluated at the same concentration but alone. Figures $4 \mathrm{~B}$ and $4 \mathrm{C}$ present the analogous relationships for perceived odor and perceived pungency, respectively. In each part of the figure, the dotted line represents the identity line, drawn at $45^{\circ}$ and with slope 1.00 , around which the experimental points should have fallen if the perceived intensity of the mixtures (whether total, odor, or pungency) had coincided with the simple sum of the perceived intensities of their components.

In the case of total nasal perceived intensity (Figure 4A), the experimental points fall around a straight line of slope 1.00 but shift downward with respect to the identity line. This means that, in the explored range, the mixtures show hypoadditivity.

Regarding odor intensity (Figure 4B), the data points cluster below the identity line, indicating hypoadditivity of odor in the mixtures. Figure 4B again points out that the range of perceived odor across all stimuli is very constricted and that the wide variation in total nasal perceived

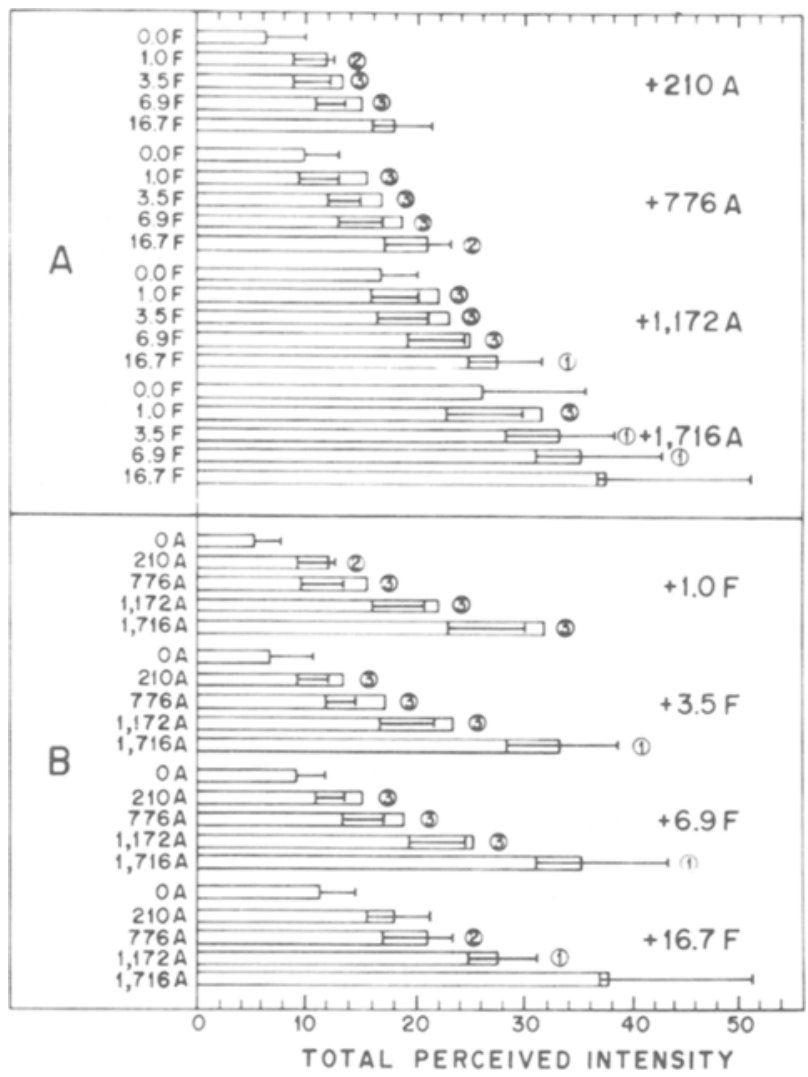

Figure 5. (A) Histogram depicting total nasal perceived intensity of the various ammonia ( $A=$ ammonia) concentrations (in ppm), alone and in the presence of various formaldehyde $(F=$ formaldehyde) backgrounds (in ppm). (B) Analogous histogram for the various formaldehyde concentrations, alone and in the presence of various ammonia backgrounds. Each bar represents the geometric mean $( \pm S E)$ of the average of two replicates made by each of 28 subjects for that stimulus. Empty rectangles at the end of the bars represent hypoadditivity degrees (see text). Bars marked with an encircled number indicate significant hypoadditivity according to (1) $p<.05$, (2) $p<.01$, (3) $p<.001$ ( $t$ test).

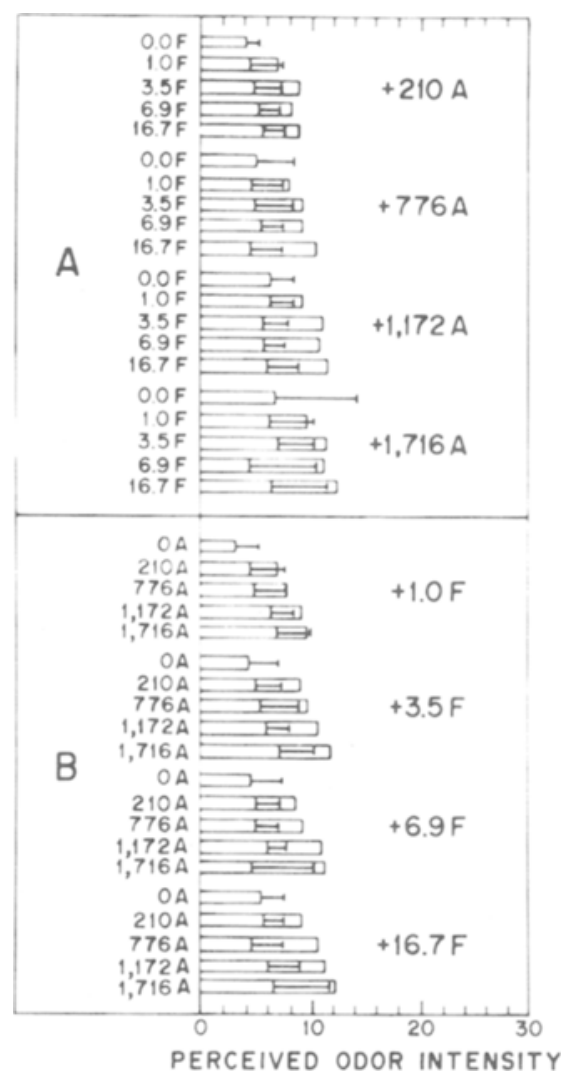

Figure 6. Same as Figure 5, but for perceived odor intensity. All bars representing mixtures of stimuli (both components different from 0 ) show significant hypoadditivity at $p<.005$ ( $t$ test).

intensity seen in Figures 1 and $4 \mathrm{~A}$ is mainly due to pungency.

When it comes to perceived pungency (Figure 4C), the experimental points obtained fall around the identity line at low and intermediate levels of pungency-indicating simple addition-but then depart from it upward, indicating a tendency to hyperaddition. A straight line of slope 1.30 fits the data reasonably well $(r=0.97)$.

Histograms illustrating the type and degree of additivity for the total nasal perceived intensity of each binary mixture can be seen in Figure 5. Figure 5A shows the total perceived intensity for each of the four ammonia concentrations in the absence and in the presence of four levels of formaldehyde. Figure 5B shows the same for the four formaldehyde concentrations in the absence and presence of ammonia. Presenting the data in this way allows a direct, single-frame comparison of the effect of the various backgrounds of each stimulus on the sensation elicited by the increasing series of the other stimulus within the referential context of what the perceived intensity would be assuming simple addition of the components. We believe that some redundance pays off in the possibility of this revealing direct visual comparison.

The bars that represent the total nasal perceived intensity of the binary mixtures carry on their right extreme a rectangle which, in Figure 5, is always empty. The 


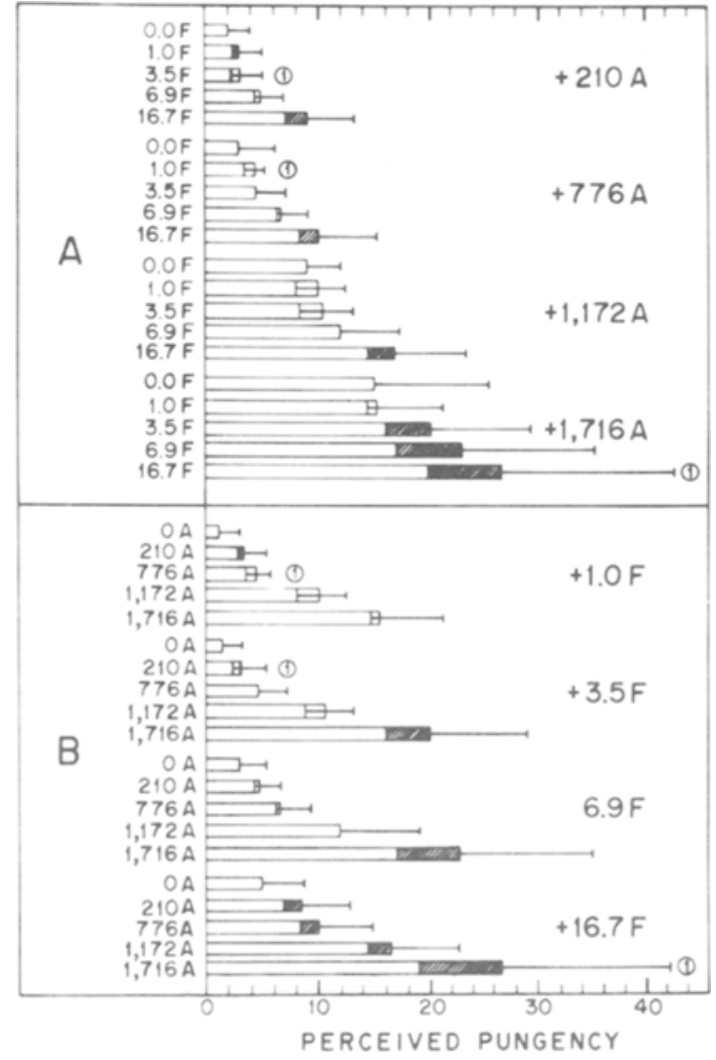

Figure 7. Same as Figure 5, but for perceived pungency. Empty rectangles at the end of the bars represent hypoadditivity degrees; shaded rectangles represent hyperadditivity degrees (see text). Bars marked with an encircled number indicate significant hypoadditivity (bars with empty rectangles) or significant hyperadditivity (bars with shaded rectangles) according to $p<.05$ ( $t$ test).

empty rectangles indicate hypoadditive mixtures. So in these cases, the extreme left portion of the rectangle, where the segment representing the standard error begins, indicates the mixture intensity. The extreme right portion of the rectangle indicates the sum of that particular mixture's component intensities when presented alone. Here, the latter sum is always significantly higher than the former mixture-intensity value, an outcome that indicates hypoaddition, except for two mixtures $(210 \mathrm{~A}+16.7 \mathrm{~F}$ and $1,716 \mathrm{~A}+16.7 \mathrm{~F}$ ), which show simple addition since there is no significant difference between the intensity values at each extreme of the rectangle.

In Figures 5, 6, and 7, we compared each mixture total, odor, or pungency intensity, respectively, with the sum of its component intensities for that same attribute (total, odor, or pungency) for each subject. The logarithm of the subjects' normalized magnitude estimations was used for the $t$ tests since such estimations show a lognormal distribution (J. C. Stevens, 1957; S. S. Stevens, 1975).

Figures 6 and 7 show histograms analogous to the one in Figure 5, but for the attributes perceived odor and perceived pungency, respectively. Note that regarding odor perception (Figure 6) all mixtures showed highly significant hypoadditivity. The picture is different for pungency (Figure 7). Here, we see some shaded rectangles, which represent hyperadditivity mixtures, at the end of the bars. The extreme left portion of the shaded rectangle now indicates the sum of the mixtures' component intensities, whereas the extreme right portion (higher intensity) indicates the mixture intensity. The outcome for pungency revealed that only two mixtures were significantly hypoadditive, 13 showed simple additivity, and one mixture, the one involving the highest concentration of each of the two stimuli, showed significant hyperadditivity.

\section{DISCUSSION AND CONCLUSIONS}

Previous data had revealed that the relation between the total nasal perceived intensity of binary mixtures of pungent odorants and that of their components was concentration-dependent (Cometto-Muñiz et al., 1989). At low concentrations, the mixtures showed hypoadditivity. This outcome progressively gave place to simple additivity at higher concentrations, and even suggested hyperadditivity at still higher stimlus levels.

The present investigation goes one step further, showing that the above-mentioned phenomenon is the result of the simultaneous activation of two sensory channelsolfaction and the CCS-with different rules of additivity for processing mixtures of stimuli. Thus, the rule of additivity for total nasal perceived intensity of the mixtures changes along the concentration range, reflecting the dominant sensation-odor or pungency-for that particular range. Odor sensations show a degree of additivity substantially lower than that of pungent sensations.

Within the frame of nasal perception of mixtures of chemicals, a question of interest explored in this investigation and not previously addressed is, How does total nasal sensation compare to odor and pungent sensations, not only in terms of how the intensity of the mixtures relates to the intensity of their components, but also in terms of stimulus-response functions?

The results presented in Figures 1, 2, and 3 clearly indicate that, in our experimental conditions, the psychophysical functions for total nasal intensity were dominated by pungency; the functions for total intensity (Figure 1) and for pungency (Figure 3) resemble each other, whereas the odor functions (Figure 2) differ. Previous studies (Cain, 1974, 1976; Cometto-Muñiz et al., 1989; J. C. Stevens \& Cain, 1986) suggested that pungency stimulus-response functions are steeper than odor stimulus-response functions. Our findings are in agreement with this suggestion, although, admittedly, the odor intensity range in our conditions was too narrow, and pungency was almost always greater than odor. A fairer test of this hypothesis should employ concentrations and chemicals in which pungency and odor were more equally matched.

Data were initially plotted in log-log coordinates to see if they could be described in terms of S. S. Stevens's 
(1957, 1975) power law relating sensation to physical stimuli. As in previous studies (Cometto-Muñiz \& Cain, 1984; Cometto-Muñiz et al., 1989), ammonia functions for both total intensity and pungency showed, in logarithmic coordinates, a consistent upward concavity. Formaldehyde functions for total intensity conformed more closely to a power function, whereas formaldehyde functions for pungency departed from a power function, also showing a tendency for upward concavity, albeit not to the extent that ammonia functions did.

At present, there is no clear-cut explanation for the upward concavity seen in some psychophysical functions. It might be that they follow a different relationship than the classical "power law." For example, in the field of taste, the Beidler equation seems to provide a good description for human taste sensations (Calviño, 1986; McBride, 1987). It might also be the case that subjects do not make a perfect discrimination between odor and pungency. Thus, at low concentrations, estimates of pungency could be "contaminated" by odor. Confusion amongst sensations elicited by different chemical senses has already been reported (Murphy \& Cain, 1980; Murphy, Cain, \& Bartoshuk, 1977). Nevertheless, a comparison of the magnitude estimations for total nasal intensity (Figure 1) and pungency (Figure 3) at the lower concentrations of both stimuli reveals that, when odor is disregarded, perceived (pungency) intensity falls dramatically near zero. This reflects the subjects' ability to ignore odor sensations even when they are proportionally stronger than pungency sensations. So, even if subjects might not be able to make a "perfect" discrimination between odor and pungency, the results show that they are able to make such a distinction reasonably well within the variability inherent to any set of biological data. In any case, failure of the subjects to differentiate "perfectly" between odor and pungency could only result in obscuring any differences in the way those sensations add up in mixtures, so our present findings, if anything, would underestimate the real differences between the way these two sensory channels process mixtures.

A model proposed for taste mixtures (Bartoshuk, 1975) states that a steeper psychophysical function leads to a higher degree of additivity in mixtures. This seems to hold for the odor and pungency of our mixtures, with pungency presenting steeper functions and a higher degree of additivity than odor.

The histograms depicted in Figures 5, 6, and 7 also point out a similarity between total intensity (Figure 5) and pungency (Figure 7), in that both show a monotonic increase in the perceived intensity of mixtures of growing concentrations of the stimuli. In contrast, the histograms representing odor (Figure 6) show this monotonic increase only in mixtures of the lower concentrations of the odorants, and even there, it is very much attenuated. In mixtures of higher concentrations, the odor intensity is arrested and ceases to augment monotonically with increasing physical levels of the odorants mixed.
Even when odor intensity is arrested in the mixtures of intermediate and high concentrations of the stimuli, total nasal and pungency intensity keep growing in those mixtures. This means that perceived pungency inhibits perceived odor, as seen in mixtures of other stimuli (Cain \& Murphy, 1980).

There is also an indication that odor might be able to inhibit pungency at low levels of pungency, as seen in Figure 7 , where the perceived pungency of a mixture of $210 \mathrm{ppm}$ ammonia and $1.0 \mathrm{ppm}$ formaldehyde fails to increase-even slightly as the odor does in Figure 6when the concentration of formaldehyde rises to $3.5 \mathrm{ppm}$. The predominance of odor at those levels and, consequently, its inhibitory influence on pungency is further supported by the fact that this mixture of $210 \mathrm{ppm} \mathrm{A} \mathrm{+}$ $3.5 \mathrm{ppm} F$ is one of the only two mixtures, out of 16 , that shows significant hypoaddition of pungency. In any case, the concentrations and stimuli employed in the present study do not allow a robust test of the possible inhibitory effect of perceived odor on low levels of perceived pungency. Using mixtures of stimuli that appeal far more to one attribute (e.g., odor) than to the other (pungency) and vice versa - as has been done before (Cain \& Murphy, 1980)-could prove crucial in the investigation of the above-mentioned inhibition. This is certainly worthy of further study.

We have mentioned that in terms of the stimulusresponse function shapes (Figures 1, 2, and 3) and the perceived-intensity histogram profiles (Figures 5, 6, and $7)$, the attributes total nasal intensity and pungency resemble each other, whereas perceived odor differs. Nevertheless, when it comes to the relationship between the perceived intensity of each mixture and that of its components, total nasal intensity and perceived odor share a feature that is not shared by perceived pungency. The former two sensations are characterized by hypoadditivity, the latter, mainly, by simple additivity.

Note that the perceived pungency of the mixtures, although roughly additive, is not perfectly so. Additivity increases with concentration. Thus, perceived pungency of mixtures of the lower concentrations of formaldehyde and ammonia shows significant hypoadditivity, whereas pungency of the mixture of the highest concentrations shows significant hyperadditivity. The remaining $13 \mathrm{mix}$ tures show simple additivity.

Degree of additivity also tends to increase for total nasal perceived intensity, in which hypoadditivity appears for 14 mixtures, but simple additivity appears for two mixtures, one of them involving the highest concentration of the stimuli employed.

More research is needed to test the possible generalization of these results to other pungent stimuli. Some investigators in the field of taste have questioned the validity of direct scaling, such as magnitude estimation, as a useful tool in understanding the additive properties of mixtures of tastants (De Graaf, Frijters, \& Van Trijp, 1987; McBride, 1986). Thus, the linearity of different scaling 
methods remains controversial. Nevertheless, we have no reason to believe that any departure from linearity present in our scaling procedure would affect judgments of odor and pungency differentially. The differences found in the way olfaction and the CCS process mixtures of stimuli would still be valid. The effects of such procedural manipulations could be better understood by testing the additive properties of the odorous and pungent sensations elicited by these same stimuli with other scaling methods and relating the outcome to the present findings.

Another way to attack this problem would be to present subjects with each single stimulus and mixture three times. In one instance, at random, they would be asked to judge total nasal perceived intensity, on another, perceived odor, and on still another, perceived pungency. Such a procedure was used in a study of the perception of different attributes of single odorants (Cain, 1976). This technique would probably call for a reduction in the number of stimuli employed, since each one would have to be presented three times and at least twice for each attribute, but another important additional insight would be gained on the interaction between odor and pungency and how they relate to total nasal sensation.

\section{REFERENCES}

BAKER, R. A. (1964). Response parameters including synergismantagonism in aqueous odor measurement. Annals of the New York Academy of Sciences, 116, 495-503.

BartoshuK, L. M. (1975). Taste mixtures: Is mixture suppression related to compression? Physiology \& Behavior, 14, 643-649.

BEIDLER, L. M. (1965). Comparison of gustatory receptors, olfactory receptors and free nerve endings. Cold Spring Harbor Symposium on Quantitative Biology, 30, 191-200.

BERGLUND, B. (1974). Quantitative and qualitative analysis of industrial odors with human observers. Annals of the New York Academy of Sciences, 237, 35-51.

Berglund, B., Berglund, U., \& Lindvall, T. (1971). On the principle of odor interaction. Acta Psychologica, 35, 255-268.

Berglund, B., Berglund, U., \& Lindvall, T. (1973). Perceptual interaction of odors from a pulp mill. Proceedings of the Third International Clean Air Congress (pp. A40-A43). Düsseldorf: VDI-Verlag.

CAIN, W. S. (1974). Perception of odor intensity and the time course of olfactory adaptation. ASHRAE Transactions, 80, 53-75.

CAIN, W. S. (1975). Odor intensity: Mixtures and masking. Chemical Senses \& Flavor, 1, 339-352.

CAIN, W. S. (1976). Olfaction and the common chemical sense: Some psychophysical contrasts. Sensory Processes, 1, 57-67.

CAIN, W. S., \& DREXLER, M. (1974). Scope and evaluation of odor counteraction and masking. Annals of the New York Academy of Sciences, 237, 427-439.

CAIN, W. S., \& Moskowitz, H. R. (1974). Psychophysical scaling of odor. In A. Turk, J. W. Johnston, Jr., \& D. G. Moulton (Eds.), Human responses to environmental odors (pp. 1-32), New York: Academic Press.

CaIN, W. S., \& MuRPhy, C. L. (1980). Interaction between chemoreceptor modalities of odour and irritation. Nature, 284, 255-257.

Calviño, A. M. (1986). Perception of sweetness: The effects of concentration and temperature. Physiology \& Behavior, 36, 1021-1028.

Cauna, N., Hinderer, K. H., \& Wentges, R. T. (1969). Sensoty receptor organs of the human nasal mucosa. American Journal of Anatomy, 124, 187-210.

Cometto-Muñiz, J. E., \& CAin, W. S. (1982). Perception of nasal pungency in smokers and nonsmokers. Physiology \& Behavior, 29, 727-731.

Cometto-Muñız, J. E., \& Cain, W. S. (1984). Temporal integration of pungency. Chemical Senses, 8, 315-327.

Cometto-Muñiz, J. E., García-Medina, M. R., Calviño, A. M. (1989). Perception of pungent odorants alone and in binary mixtures. Chemical Senses, 14, 163-173.

Cometto-Muñiz, J. E., García-Medina, M. R., Calviño, A. M., \& NoriegA, G. (1987). Interactions between $\mathrm{CO}_{2}$ oral pungency and taste. Perception, 16, 629-640.

Cometro-Muñiz, J. E., Noriega, G. (1985). Gender differences in the perception of pungency. Physiology \& Behavior, 34, 385-389.

CowART, B. (1987). Oral chemical irritation: Does it suppress taste intensity? Chemical Senses, 12, 467-479.

De Graaf, C., Frijters, J. E. R., \& van Trijp, H. C. M. (1987). Taste interaction between glucose and fructose assessed by functional measurement. Perception \& Psychophysics, 41, 383-392.

Doty, R. L. (1975). Intranasal trigeminal detection of chemical vapors by humans. Physiology \& Behavior, 14, 855-859.

Doty, R. L., Brugger, W. E., Jurs, P. C., Orndorff, M. A., SNYDER, P. J., \& LowRY, L. D. (1978). Intranasal trigeminal stimulation from odorous volatiles: Psychometric responses from anosmic and normal humans. Physiology \& Behavior, 20, 175-185.

Dunér-Engström, M., Fredholm, B. B., Larsson, O., Lundherg, J. M., \& SARIA, A. (1986). Autonomic mechanisms underlying capsaicin induced oral sensations and salivation in man. Journal of Physiology, 373, 87-96.

Dunn, J. D., Cometto-Muñız, J. E., \& Cain, W. S. (1982). Nasal reflexes: Reduced sensitivity to $\mathrm{CO}_{2}$ irritation in cigarette smokers. Joumal of Applied Toxicology, 2, 176-178.

Foster, D. (1963). Odors in series and parallel. Proceedings of the Scientific Section, Toilet Goods Association, 39, 1-6.

García-Medina, M. R., \& Cain, W. S. (1982). Bilateral integration in the common chemical sense. Physiology \& Behavior, 29, 349-353.

GREEN, B. G. (1986). Sensory interactions between capsaicin and temperature in the oral cavity. Chemical Senses, 11, 371-382.

JoNES, F. N., Woskow, M. H. (1964). On the intensity of odor mixtures. Annals of the New York Academy of Sciences, 116, 484-494.

KOSTER, E. P. (1969). Intensity in mixtures of odorous substances. In C. Pfaffman (Ed.), Olfaction and taste (Vol. 3, pp. 142-149). New York: Rockefeller University Press.

LAFFORT, P., \& DRAVNiEks, A. (1982). Several models of suprathreshold quantitative olfactory interaction in humans applied to binary, ternary and quaternary mixtures. Chemical Senses, 7, 153-174.

Laing, D. G., Panhuber, H., Willcox, M. E., \&ittman, E. A. (1984). Quality and intensity of binary odor mixtures. Physiology \& Behavior, 33, 309-319.

Laing, D. G., Willcox, M. E. (1983). Perception of components in binary odour mixtures. Chemical Senses, 7, 249-264.

Lane, H. L., Catania, A. C., \& Stevens, S. S. (1961). Voice level: Autophonic scale, perceived loudness and effects of sidetone. Journal of the Acoustical Society of America, 33, 160-167.

LAwLESS, H. (1984). Oral chemical irritation: Psychophysical properties. Chemical Senses, 9, 143-155.

LAWLESS, H. T. (1987). An olfactory analogy to release from mixture suppression in taste. Bulletin of the Psychonomic Society, 25, 266-268.

Lawless, H., Rozin, P., Shenker, J. (1985). Effects of oral capsaicin on gustatory, olfactory and irritant sensations and flavor identification in humans who regularly or rarely consume chili pepper. Chemical Senses, 10, 579-589.

LAwless, H., \& Stevens, D. A. (1984). Effects of oral chemical irritation on taste. Physiology \& Behavior, 32, 995-998.

Lawless, H. T. \& Stevens, D. A. (1988). Responses by humans to oral chemical irritants as a function of locus of stimulation. Perception \& Psychophysics, 43, 72-78.

MCBRIDE, R. L. (1986). The sweetness of binary mixtures of sucrose, fructose and glucose. Journal of Experimental Psychology: Human Perception \& Performance. 12, 584-594.

McBride, R. L., (1987). Taste psychophysics and the Beidler equation. Chemical Senses, 12, 323-332. 
Moskowitz, H. R., \& Barbe, C. D. (1977). Profiling of odor components and their mixtures. Sensory Processes, 1, 212-226.

MURPHY, C. (1983). Age-related effects on the threshold, psychophysical function, and pleasantness of menthol. Journal of Gerontology, $\mathbf{3 8}$, 217-222.

MuRPhy, C., \& CAIN, W. S. (1980). Taste and olfaction: Independence vs interaction. Physiology \& Behavior, 24, 601-605.

Murphy, C., Cain, W. S., \& Bartoshuk, L. M. (1977). Mutual action of taste and olfaction. Sensory Processes, 1, 204-211.

National Institute for Occupational Safety and Health (NIOSH) (1973). Manual of analytical methods (2nd ed., Vol. 1). Washington, DC: U.S. Department of Health, Education and Welfare.

National Institute for Occupational Safety and Health (NIOSH) (1974). Criteria for a recommended standard: Occupational exposure to ammonia. Washington, DC: U.S. Department of Health, Education and Welfare.

PATte, F., \& LAFfort, P. (1979). An alternative model of olfactory quantitative interaction in binary mixtures. Chemical Senses \& Flavor, 4, 267-274.

Rosen, A. A., Peter, J. B., \& Middleton, F. M. (1962). Odor threshold of mixed organic chemicals. Journal of the Water Pollution Control Federation, 35, 7-14.

Rozin, P., Ebert, L., \& SChull, J. (1982). Some like it hot: A temporal analysis of hedonic responses to chili pepper. Apetite, 3, 13-22.

Rozin, P., Mark, M., \& SChILlek, D. (1981). The role of desensitization to capsaicin in chili pepper ingestion and preference. Chemical Senses, 6, 23-31.
Rozin, P., \& SCHILler, D. (1980). The nature and acquisition of a preference for chili pepper by humans. Motivation \& Emotion, 4. 77-101.

SIZER, F., Harrus, N. (1985). The influence of common food additives and temperature on threshold perception of capsaicin. Chemical Senses, 10, 279-286.

Stevens, D. A., Lawless, H. T. (1986). Putting out the fire: Effects of tastants on oral chemical irritation. Perception \& Psychophysics, 39, 346-350.

STEVENS, J. C. (1957). A comparison of ratio scales for the loudness of white noise and the brightness of white light. Unpublished doctoral dissertation, Harvard University, Cambridge, MA.

Stevens, J. C., Cain, W. S. (1986). Aging and the perception of nasal irritation. Physiology \& Behavior, 37, 323-328.

Stevens, J. C., \& MaRks, L. E. (1980). Cross-modality functions generated by magnitude estimation. Perception \& Psychophysics, 27, 379-389.

Stevens, S. S. (1957). On the psychophysical law. Psychological Review, 64, 153-181.

Stevens, S. S. (1975), Psychophysics: Introduction to its perceptual, neural and social prospects. New York: Wiley.

ZwaARdemaker, H. (1907). Über die Proportionen der Geruchs Kompensation. Archiv fur Anatomie und Physiologie, 31(Suppl), 59-70.

(Manuscript received July 17, 1989; revision accepted for publication October 25, 1989.) 